Proyecciones

Vol. 18, No 2, pp. 165-173, December 1999

Universidad Católica del Norte

Antofagasta - Chile

\title{
A NOTE ON THE HOMOLOGY COVERING OF CLOSED KLEIN SURFACES *
}

\author{
RUBEN HIDALGO \\ Universidad Técnica Federico Santa María, Valparaíso- Chile
}

\begin{abstract}
In previous works we have seen that a finitely generated torsionfree non-elementary function group is uniquely determined by its commutator subgroup. In this note, we observe that under the presence of orientation-reversing conformal automorphisms the above rigidity property still valid. More precisely, we see that finitely generated torsion-free reversing Fuchsian groups of the first kind, without parabolic transformations, are uniquely determined by their commutator subgroup. The arguments of the proof follous the same lines as for the orientable situation.
\end{abstract}

SUBJCLASS : Primary 30F20 30F45.

KEY WORDS : Klein surfaces, Homology coverings.

${ }^{*}$ This paper was partially supported by projects UTFSM 991223, Fondecyt 8970007 and Presidential Science Chair on Geometry. 


\section{Introduction}

A classical result in the theory of Riemann surfaces is Torelli's theorem. Roughly speaking, this asserts that each closed Riemann surface is uniquely determined, up to conformal equivalence, by its Jacobian. More precisely, assume that $S$ is a closed Riemann surface of genus $g$, and let $\mathrm{H}^{1,0}(S)$ be the complex vector space (of dimension $g$ ) of holomorphic 1 -form on $S$. Integration of 1-forms on 1-cycles permits us to see $\mathrm{H}_{1}(S, \mathbf{Z})$ as a lattice in the dual space $\left(\mathrm{H}^{1,0}(S)\right)^{*}$ of $\mathrm{H}^{1,0}(S)$. The quotient $J(S)=\left(\mathrm{H}^{1,0}(S)\right)^{*} / \mathrm{H}_{1}(S, \mathbf{Z})$ is a complex torus of dimension $g$, called the Jacobian of $S$. Torelli's theorem asserts that the complex structure of $J(S)$ (together a special divisor) determines the complex structure of $S$ up to conformal equivalence.

If we choose a point $p_{0} \in S$, we have a natural holomorphic embedding (see [1]) $\Phi_{p_{0}}: S \hookrightarrow J(S)$, defined by $\Phi_{p_{0}}(p)=\left[\int_{p_{0}}^{p}\right]$. If we denote by $\pi$ : $\left(\mathrm{H}^{1,0}(S)\right)^{*} \rightarrow J(S)$ the universal covering induced by the action of $\mathrm{H}_{1}(S, \mathbf{Z})$, then we can lift $\Phi_{p_{0}}(S) \cong S$ to obtain a Riemann surface $\widetilde{S} \subset\left(\mathrm{H}^{1,0}(S)\right)^{*}$ with $\mathrm{H}_{1}(S, \mathbf{Z})$ acting as group of conformal automorphisms and so that $\widetilde{S} / \mathrm{H}_{1}(S, \mathbf{Z})=S$. The surface $\widetilde{S}$ is the homological cover of $S$.

If $F$ is a Fuchsian group that uniformizes $S$, then $[F, F]$, the commutator subgroup of $F$, uniformizes $\widetilde{S}$. In [4] B. Maskit has shown an equivalent to Torelli's theorem for Fuchsian groups, that is, that $[F, F]$ determines uniquely $F$ and, in particular, that the homological cover of a closed Riemann surface determines it uniquely up to conformal equivalence. It is not clear yet if one can obtain Torelli's theorem from Maskit's result or vice-versa.

A reversing Fuchsian group is by definition a discrete group of confor$\mathrm{mal} /$ anticonformal automorphisms of the Riemann sphere that keeps invariant some euclidean disc (our definition is different as done by some authors that obligate a reversing Fuchsian group to have orientation-reversing transformations). The boundary circle contains the limit set of this group. If this limit set is all this circle, then we say that the group is of first kind; otherwise, is called of second kind. The index two subgroup of orientationpreserving transformations, if the group has orientation-reversing transformations, is a Fuchsian group of the same kind.

If $G$ is a finitely generated torsion-free reversing Fuchsian group of the first kind, without parabolic transformations, keeping invariant the euclidean disc $\Delta$, then $S=\Delta / G$ is a closed Klein surface. The homology cover of $S$ is a usual Riemann surface (that is, orientable Klein surface) and it is conformally equivalent to $\Delta /[G, G]$, where $[G, G]$ denotes the commutator 
subgroup of $G$. The main result of this note is the following.

Theorem 1. Let $G$ be a finitely generated torsion-free reversing Fuchsian group of the first kind. If $G$ has no parabolic transformations, then its commutator $[G, G]$ determines $G$ uniquely.

Corollary 1. Let $S$ be a closed Klein surface of genus $\gamma \geq 2$. Then the Riemann surface structure of the homology cover of $S$ determines, up to di-analytic equivalence, the Klein structure of $S$.

Proof. Assume we have two closed Klein surfaces $S_{1}$ and $S_{2}$, both of hyperbolic type, with biholomorphic homology covers. We uniformize each $S_{i}$ by a reversing Fuchsian group $F_{i}$ acting on the unit disc $D$. Since the homology covers are conformally equivalent, we may assume $\left[F_{1}, F_{1}\right]=$ $\left[F_{2}, F_{2}\right]$. It follows from theorem 1 that $F_{1}=F_{2}$.

In the presence of parabolic transformations, theorem 1 still valid if there is no orientation-reversing transformation [2], [3]. We do not know at the present time if theoren 1 holds in the presence of parabolic and reversing-orientation transformations.

Theoren 1 combined with the Riemann map's theorem gives us the following.

Theorem 2. Let $G$ be a finitely generated torsion-free non-elementary kleinian group of conformal/anticonformal automorphisms of the Riemann sphere for which there is a simply connected invariant component of its region of discontinuity. If $G$ has no parabolic transformations, then its commutator $[G, G]$ determines it uniquely.

Proof. Let us assume we have $G_{1}$ and $G_{2}$ kleinian groups of confor$\mathrm{mal} /$ anticonformal automorphisms, each one keeping invariant a simply connected component of their respective region of discontinuity and having the same commutator subgroup $K$. Let us denote by $\Delta_{1}$ (respectively, $\Delta_{2}$ ) such an invariant component of $G_{1}$ (respectively, $G_{2}$ ). Let us consider the case that $\Delta_{1} \neq \Delta_{2}$. The commutator subgroup $K$ is normal subgroup in each $G_{i}$. It follows that the region of discontinuity of $G_{1}, G_{2}$ and $K$ is the same, say $\Omega$. If $\Delta_{1}$ is not invariant under $G_{2}$, then there is some $g \in G_{2}$ so that $g\left(\Delta_{1}\right) \neq \Delta_{1}$. Since $K$ is a normal subgroup of $G_{2}, K$ also keeps invariant $g\left(\Delta_{1}\right)$. Now, since $g\left(\Delta_{2}\right)=\Delta_{2}$, we have that the region of 
discontinuity $\Omega$ has three different simply connected components, each one invariant under $K$, a contradiction. As a consequence, we may assume that both $G_{1}$ and $G_{2}$ have the same simply connected invariant component, say $\Delta$. Use a Riemann map $w: D \rightarrow \Delta$, where $D$ is the unit disc. Then the groups $F_{i}=w^{-1} G_{i} w, i=1,2$, are torsion-free reversing Fuchsian groups of the first kind without parabolic transformations (to have a parabolic transformation in the Fuchsian group $F_{i}$ is necessary, but not sufficient, to have parabolic transformations in $G_{i}$ ) with common commutator subgroup. The result now follows from theorem 1 .

\section{Klein Surfaces}

A Kilcin surface is a Hausdorff topological space with a system of coordinates $\left\{\left(U_{\alpha}, z_{\alpha}\right): \alpha \in A\right\}$, so that $z_{\alpha}\left(U_{\alpha}\right)=V_{\alpha}$ is an open set in the closed half-plane $\overline{\mathbf{H}}=\{z \in \mathbf{C}: \operatorname{Im}(z) \geq 0\}$, so that change of coordinates are either analytic or anti-analytic maps. Set $\mathbf{H}=\{z \in \mathbf{C}: \operatorname{Im}(z)>0\}$ the hyperbolic half-plane.

If we have that $V_{\alpha} \subset \mathbf{H}$ for all $\alpha$, then we say that the Klein surface has no boundary. The points in the surface corresponding to $V_{\alpha} \cap \partial(\mathbf{H})$ are the boundary points; the boundary of the Klein surface is the subset formed by the boundary points.

A Riemann surface is a Klein surface for which all change of coordinates are analytic. If the Klein surface is compact without boundary, then we say that it is closed.

A closed Riemann surface homeomorphic to the connected sum of $g \geq 1$ tori is said to have genus $g$. If it is homeomorphic to the sphere, then it has genus 0 .

A non-orientable closed Klein surface $S$ is said to have genus $g \geq 1$ if it is homeomorphic to the connected sum of $g$ real projective planes. In this case: if $g=2 p+2$, then $S$ is homeomorphic to the connected sum of a closed orientable surface of genus $p$ and two projective planes, and if $g=2 p+1$, then $S$ is homeomorphic to the connected sum of a closed orientable surface of genus $p$ and a projective plane.

A closed Klein surface is uniformized by a reversing Fuchsian group of the first kind if and only if the genus is greater or equal to two for the orientable case and greater or equal to three in the non-orientable case. In that case, we say that the surface is of hyperbolic type.

If $S$ is a closed Klein surface, then its first homology group with real coefficients $\mathrm{H}_{1}(S, \mathbf{R})$ is exactly the tensor product $\mathrm{H}_{1}(S, \mathbf{Z})_{f} \otimes \mathbf{R}$, where 
$\mathrm{H}_{1}(S, \mathbf{Z})_{f}$ is the free part of $\mathrm{H}_{1}(S, \mathbf{Z})$. In particular, (a) if $S$ is a closed Riemam surface of genus $g$, then $H_{1}(S, \mathbf{R})$ is real isomorphic to $\mathbf{R}^{2 g}$; (b) if $S$ is a non-orientable closed Klein surface of genus $g$, then $H_{1}(S, \mathbf{R})$ is isomorphic to $\mathbf{R}^{g-1}$.

A Klein surface $\widehat{S}$ is an abelian cover of $S$ if there exists a regular dianalytic covering $\pi: \widehat{S} \rightarrow S$ with abelian group of deck transformations. The homology covering of $S, \pi: \widetilde{S} \rightarrow S$, is the highest abelian covering of $S$, that is. it is the covering corresponding to the commutator subgroup of the fundamental group $\Pi_{1}(S)$ of $S$. It follows that the homology cover $\widetilde{S}$ of $S$ is necessarily a Riemann surface.

\section{Harmonic Differential Forms on Klein Surfaces}

The proof of theorem 1 involves harmonic differential forms on Klein surfaces.

I.- Let us assume $X$ is a closed Klein surfaces of genus $g \geq 2$, and denote by $\mathrm{H}^{1}(X)$ the real vector space generated by the harmonic real 1-forms on $X$.

Riemann-Roch theorem asserts that if $X$ is a closed Riemann surface of genus $g$, then $\operatorname{dim}\left(\mathrm{H}^{1}(X)\right)=2 g$.

For non-orientable closed Klein surfaces, the dimension of $\mathrm{H}^{1}(X)$ is obtained as follows. We consider the double orientable cover $\tilde{X}$ of $X$, which is a closed Riemann surface of genus $g-1$. The Klein surface $X$ is obtained as quotient of $\tilde{X}$ by an anticonformal involution $\tau$ acting free fixed points on $X$.

The space $H^{1}(X)$ can be identified with the real vector space associated to the eigenvalue +1 of the real linear transformation induced by $\tau$. This together the Rienann-Roch theorem gives that $\operatorname{dim}\left(\mathrm{H}^{1}(X)\right)=g-1$.

In both cases, orientable and non-orientable, there is a duality between the spaces $\mathrm{H}^{1}(X)$ and $\mathrm{H}_{1}(X, \mathbf{R})$ given by integration of 1 -forms on cycles. In fact, for the orientable case, it is proved in [1]. For the non-orientable case, we use the double covering $P: \widetilde{X} \rightarrow X$. In this case, we have natural

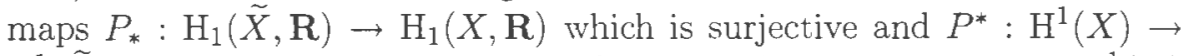
$\mathrm{H}^{1}(\tilde{X})$ which is one-to-one, so that $\int_{\alpha} P^{*} w=\int_{P_{*}(\alpha)} w$, for all $w \in \mathrm{H}_{1}^{1}(X)$ and all $\alpha \in \mathrm{H}_{1}(\tilde{X}, \mathbf{R})$. In this case, $P^{*}\left(\mathrm{H}^{1}(X)\right)$ consists exactly of those 1 -forms in $\mathrm{H}^{1}(\tilde{X})$ invariant under the action of $\tau^{*}$ (the action of pull-backs forms by $\tau$ ). Then the duality, by integration of forms on cycles, between $\mathrm{H}_{1}(\widetilde{X}, \mathbf{R})$ and $\mathrm{H}^{1}(\widetilde{X})$ implies the desired duality between $\mathrm{H}_{1}(X, \mathbf{R})$ and $\mathrm{H}^{1}(X)$. 
II.- Let $X$ be a compact Klein surface with boundary. Assume $X$ has genus $\gamma$, and $h$ boundary components. We may consider a closed Riemann surface $X^{d}$ of genus $g=\gamma+h-1$, having an anticonformal involution $\tau: X^{d} \rightarrow X^{d}$ so that $X^{d} / \tau=X$, with exactly $h$ connected components of fixed points.

We consider $\tau^{*}$, the natural action (by pull-back) of $\tau$ on the real vector space $\mathrm{H}^{1}\left(X^{d}\right)$. The subvector space given by the fixed points of $\tau^{*}$ is identified as $\mathrm{H}^{1}(X)$. In this case, as a consequence of the Riemann-Roch theorem, we get $\operatorname{dim}\left(H^{1}(X)\right)=\gamma+h-1$.

\section{The Proof of Theorem 1}

Let us start with two finitely generated torsion-free reversing Fuchsian groups, without parabolic transformations, of the first kind, say $F_{1}$ and $F_{2}$, acting on the half-plane $\mathbf{H}$.

We set $S_{m}$ the closed Klein surface uniformized by $F_{m}$, that is, $S_{m}=$ $\mathbf{H} / F_{m}$.

We assume $F_{1}$ and $F_{2}$ to have the same commutator subgroup, that is, $\left[F_{1}, F_{1}\right]=\left[F_{2}, F_{2}\right]=K$. Set $\widetilde{S}=\mathbf{H} / K$.

We have that $\widetilde{S}$ is in fact a Riemann surface. Let us denote by $\Gamma_{m}=$ $F_{m} / K$ be the group of deck transformations for the natural cover $\pi_{m}: \widetilde{S} \rightarrow$ $S_{m}$; that is, $\widetilde{S} / \Gamma_{m}=S_{m}$. We need to show that $\Gamma_{1}=\Gamma_{2}$.

It is well known that $\widetilde{S}$ is conformally equivalent to none of the following Riemann surfaces: (i) The Riemann sphere, (ii) the complex plane, (iii) the complex plane with one point deleted, (iv) a torus, (v) the unit disk, and (vi) an annulus. Hence the full group of conformal/anticonformal automorphisms of $\widetilde{S}$ acts discontinuously on $\widetilde{S}$; in particular, the group $\Gamma$ of automorphisms of $\widetilde{S}$ generated by $\Gamma_{1}$ and $\Gamma_{2}$ acts discontinuously on $\widetilde{S}$. Set $S=\widetilde{S} / \Gamma$.

Since $S_{1}$ and $S_{2}$ are closed Klein surfaces of hyperbolic type, they have finite hyperbolic area. Hence they are finite sheeted coverings of $S$; that is, both $\Gamma_{1}$ and $\Gamma_{2}$ have finite index in $\Gamma$. It follows that $\bar{\Gamma}=\Gamma_{1} \cap \Gamma_{2}$ has finite index in $\Gamma_{1}, \Gamma_{2}$ and $\Gamma$.

Set $\bar{S}=\widetilde{S} /\left(\Gamma_{1} \cap \Gamma_{2}\right)$. We have the following commutative diagram of (branched) coverings: 


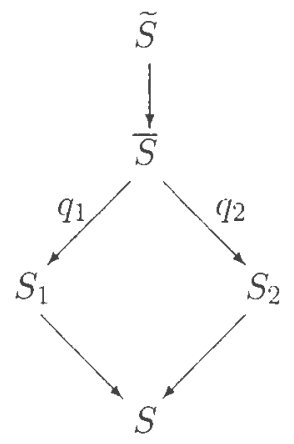

The covering $q_{m}: \bar{S} \rightarrow S_{m}$ is a regular di-analytic covering with an abelian group of deck transformations $G_{m}=\Gamma_{m} / \bar{\Gamma}$. We note that $q_{m}$ : $\bar{S} \rightarrow S_{m}$ can be extended to a di-analytic mapping of the Klein surface $\bar{S} \cup\{$ the punctures of $\bar{S}\}$ onto the Klein surface $S_{m} \cup\left\{\right.$ the punctures of $\left.S_{m}\right\}$. Set $G=\Gamma / \bar{\Gamma}$. It is enough now to show that $G_{1}=G_{2}$.

Observe that a loop $w$ on $\bar{S}$ lifts to a loop on $\widetilde{S}$ if and only if its projection to $S_{m}$ is homologically trivial. Let $\left(q_{m}\right)_{*}: \mathrm{H}_{1}(\bar{S}, \mathbf{R}) \rightarrow \mathrm{H}_{1}\left(S_{m}, \mathbf{R}\right)$ be the induced map on the first homology group with real coefficients. Since $q_{m}$ is a finite degree covering map, the map $\left(q_{m}\right)_{*}$ is onto. The above observation asserts that $\left(q_{1}\right)_{*}$ and $\left(q_{2}\right)_{*}$ both have the same kernel. It follows from this that $\mathrm{H}_{1}\left(S_{1}, \mathbf{R}\right)$ and $\mathrm{H}_{1}\left(S_{2}, \mathbf{R}\right)$ both have the same dimension.

We have natural linear injections, duals to the maps $\left(q_{m}\right)_{*}: \mathrm{H}_{1}(\bar{S}, \mathbf{R}) \rightarrow$ $\mathrm{H}_{1}\left(S_{m}, \mathbf{R}\right)$, say $q_{m}^{*}: \mathrm{H}^{1}\left(S_{m}\right) \rightarrow \mathrm{H}^{1}(\bar{S})$.

We can regard the image $q_{m}^{*}\left(\mathrm{H}^{1}\left(S_{m}\right)\right)$ as those 1-forms in $\mathrm{H}^{1}(\bar{S})$ which are invariant under the action of $G_{m}$.

The duality asserts that $q_{m}^{*}\left(\mathrm{H}^{1}\left(S_{m}\right)\right)$ is orthogonal to the kernel of $\left(q_{m}\right)_{*}$, for $m=1,2$.

The equality of the kernel of both $\left(q_{1}\right)_{*}$ and $\left(q_{2}\right)_{*}$ asserts the following equalities:

$(*) \quad \operatorname{dim}\left(\mathrm{H}^{1}\left(S_{1}\right)\right)=\operatorname{dim}\left(\mathrm{H}^{1}\left(S_{2}\right)\right)=\operatorname{dim}\left(\mathrm{H}^{1}(S)\right)$.

Let us denote by $g_{m}$ and $\gamma$ the genus of $S_{m}$ and $S$, respectively. The number of boundary components of $S$ is denoted by $h \geq 0$.

\subsection{The Case $h=0$}

Using the fact that $\operatorname{Area}\left(S_{m}\right)=d_{m} \operatorname{Area}(S)$, where $d_{m}$ is the degree of the (branched) di-analytic covering $p_{m}: S_{m} \rightarrow S$, we have the following facts: 
(i) If some $S_{m}$ is non-orientable, then $S$ is non-orientable.

(ii) $d_{m}(\gamma-2)=g_{m}-2$, if both $S_{m}$ and $S$ are non-orientable.

(ii) $d_{m}(\gamma-1)=g_{m}-1$, if both $S_{m}$ and $S$ are orientable.

(iii) $2(g-1)=d_{m}(\gamma-2)$, if $S_{m}$ is orientable and $S$ is non-orientable.

(1) Assume all three Klein surfaces are non-orientable. We have from $(*)$ that $g_{m}=\gamma$. It follows that $d_{m}=1$ and, in particular, that each group $G_{m}$ has index one in $G$. It follows that, $G_{1}=G_{2}$.

(2) If both surfaces $S_{1}$ and $S_{2}$ are orientable, then $S$ must be also orientable. In fact, both groups $F_{1}$ and $F_{2}$ have no reversing-orientation transformations. Then neither does the group generated by those two groups. ¿From (*) we have $g_{m}=\gamma$. It follows that $d_{m}=1$ and that each group $G_{m}$ has index one in $G$. It follows that, $G_{1}=G_{2}$.

(3) If $S_{1}$ is orientable and $S_{2}$ is non-orientable, then $S$ is necessarily nonorientable. In particular, $d_{1} \geq 2$. We have from $(*)$ that $2 g_{1}=g_{2}=\gamma$. From this we obtain that $-1=\left(d_{1}-1\right)(\gamma-2)$, contradicting the fact that $\gamma \geq 2$ by hyperbolicity property.

\subsection{The Case $h \geq 1$}

In this case, if both $S_{1}$ and $S_{2}$ are orientable, then $F_{1}$ and $F_{2}$ have no orientation-reversing transformations and $S$ is orientable without holes (because the group generated by $F_{1}$ and $F_{2}$ is of the first kind). This contradicts the condition $h>0$.

In particular, the condition $h>0$ obligates for either $S_{1}$ or $S_{2}$ to be a closed no-orientable surface (we may assume $S_{2}$ to be non-orientable) and $S$ to be non-orientable.

Since Area $\left(S_{m}\right)=d_{m} \operatorname{Area}(S)$, where $d_{m}$ is the degree of $p_{m}: S_{m} \rightarrow S$, we obtain:

(i) $g_{m}-2=d_{m}(\gamma+h-2)$, if $S_{m}$ is non-orientable.

(ii) $d_{m}(\gamma+h-2)=2\left(g_{m}-1\right)$, if $S_{m}$ is orientable. 
Assume that $S_{1}$ is non-orientable. The equality $(*)$ asserts that $g_{1}=$ $g_{2}=\gamma+h=t>2$ (hyperbolicity condition). From (i) we have $(t-2)\left(d_{m}-\right.$ 1) $=0$ and, in particular, $d_{m}=1$, that is, $G_{1}=G_{2}$.

Assume that $S_{1}$ is orientable, in which case $d_{1}>1$. Equality $(*)$ asserts that $\gamma+h-1=g_{2}-1=2 g_{1}=t \geq 4$ (hyperbolicity condition). From (ii) we get $\left(d_{1}-1\right)(t-1)+1=0$ a contradiction.

\section{References}

[1] H.Farkas and I. Kra. Riemann Surfaces. Graduate Texts in Mathematics, Springer-Verlag, Berlin (1980).

[2] R.A. Hidalgo. Homology coverings of Riemann surfaces. Tôhoku Math. J. 45, pp. 499-503, (1993).

[3] . A conmmutator rigidity for function groups. Preprint.

[4] B. Maskit. The Homology Covering of a Riemann Surface. Tôhoku Math. J. 38, pp. 561-562, (1986).

[5] . On boundaries of Teichmüller spaces and kleinian groups II. Ann. of Math. 91, PP. 607-639, (1970).

Received : June 10, 1998

\section{Rubén Hidalgo}

Departamento de Matemáticas

Universidad Técnica Federico Santa María

Casilla 110-V

Valparaíso

Chile 